\title{
Toward Understanding the Ionization of Biomarkers from Micrometer Particles by Bio-Aerosol Mass Spectrometry
}

\author{
Scott C. Russell, Gregg Czerwieniec, and Carlito Lebrilla \\ Department of Chemistry, University of California, Davis, California, USA
}

\author{
Herbert Tobias, David P. Fergenson, Paul Steele, Maurice Pitesky, \\ Joanne Horn, Abneesh Srivastava, Matthias Frank, and Eric E. Gard \\ Lawrence Livermore National Laboratory, Livermore, California, USA
}

\begin{abstract}
The appearance of informative signals in the mass spectra of laser-ablated bio-aerosol particles depends on the effective ionization probabilities (EIP) of individual components during the laser ionization process. This study investigates how bio-aerosol chemical composition governs the EIP values of specific components and the overall features of the spectra from the bio-aerosol mass spectrometry (BAMS). EIP values were determined for a series of amino acid, dipicolinic acid, and peptide aerosol particles to determine what chemical features aid in ionization. The spectra of individual amino acids and dipicolinic acid, as well as mixtures, were examined for extent of fragmentation and the presence of molecular ion dimers, which are indicative of ionization conditions. Standard mixtures yielded information with respect to the significance of secondary ion plume reactions on observed spectra. A greater understanding of how these parameters affect EIP and spectra characteristics of bio-aerosols will aid in the intelligent selection of viable future biomarkers for the identification of bio-terrorism agents. (J Am Soc Mass Spectrom 2004, 15, 900-909) (c) 2004 American Society for Mass Spectrometry
\end{abstract}

$\mathrm{T}$ The ability to perform species level detection of individual cells or spores, or monitor specific biological processes by mass spectrometry, requires the ionization and identification of specific markers (biomarkers). Many mass spectrometry techniques have been employed for the ionization of biomarkers directly from microorganisms, including pyrolysis electron impact mass spectrometry and fast atom bombardment [1-3]. More recently, electrospray ionization (ESI) and matrix-assisted laser desorption/ionization (MALDI) have been employed to ionize and characterize protein biomarkers for viruses, bacteria, and fungi [4-8]. Electrospray has the advantage of being a relatively soft ionization method producing multiply charged molecular ions. However, the complexity of the spectra produced from biological samples often hinders rapid interpretation [4]. Although MALDI is not hindered by such problems and has shown the ability for species level detection, it does require sample preparation with an often acidic denaturing matrix, which leads to increased analysis time as well as an additional

Published online April 9, 2004

Address reprint requests to Dr. C. B. Lebrilla, Department of Chemistry, University of California at Davis, One Shields Avenue, Davis, CA 95616, USA. E-mail: cblebrilla@ucdavis.edu degree of analysis variability [9]. The removal of sample preparation was proven successful using matrix-free laser desorption/ionization coupled with a TOF analyzer to discriminate various Bacillus species from one another [9]. However, this method still maintained the disadvantage of analyzing colonies of cells on a surface, not single cells in the atmosphere.

The current approach involves bio-aerosol mass spectrometry (BAMS), which is a method for real-time analysis of bio-aerosols [10]. Unlike traditional chemical and biochemical methods for bacteria identification, which have relatively long analysis times ranging from hours to days [11], bio-aerosol mass spectrometry requires only seconds $[10,11]$. Each particle is analyzed individually with no required sample preparation. Consequently, background particles do not complicate the analysis as they have mass spectra that are distinctly different from specific bio-particles. This technology has many potential applications including real-time screening of mail, passengers, and luggage for chemical and biological agents. One could also track the trajectories of a bio-aerosol release in order to inform and protect those most at risk of exposure. Advances in basic science may also be possible with this methodology, including the identification of biomarkers for metabolic processes, and diseases such as tuberculosis. 
Monitoring biomarkers for stages of cell development and death, i.e., a healthy cell becoming cancerous, may also be possible.

The instrument has previously been shown to identify Bacillus spores in real-time [10]. This ability is based on the use of identifying a representative fingerprint spectrum of the spore [10]. The fingerprint spectrum contains specific biomarker peaks for Bacillus spores. Dipicolinic acid, the most abundant chemical component of Bacillus spores at 5 to $14 \%$ of the dry mass, is located in the spore core [12]. Two peaks in the negative mass spectrum of Bacillus spores at $\mathrm{m} / \mathrm{z}-166.0$ and -167.0 have been identified by stable isotope labeling as deprotonated and odd electron DPA molecular ions, respectively [13]. Two additional spore core components, arginine and glutamic acid, have also been identified and are observed in the negative mass spectrum as deprotonated species at $m / z-173$ and -146 , respectively [13].

This study is focused on understanding the factors governing efficient ionization of bio-aerosols. This will help explain why only core components are observed in the Bacillus spore mass spectra. Many MALDI experiments have shown the importance of an efficient absorbing matrix to aid in the ionization process of aerosols [14-19]. Secondary plume reactions such as proton transfer reactions have also been shown to be significant in determining what ions are observed [2022]. Absorption efficiency and plume reactions likely govern the observed spectra of spores as well. Because the current approach to biomarker detection involves reagentless BAMS, the effects of particle chemical composition, without a matrix, on effective ionization probability (see the Experimental section) and observed spectra were examined with various model compounds. Model compounds were chosen which are commonly found in bacterial spores, including amino acids, peptides, and dipicolinic acid (DPA).

\section{Experimental}

\section{Aerosol Generation}

Amino acid, DPA, and mixture aerosols were generated from a Collison nebulizer (TSI, Incorporated, Shoreview, MN). Standards for the nebulizer were prepared as $2 \times 10^{-3} \mathrm{M}$ solutions in $\mathrm{H}_{2} \mathrm{O}$. All standard mixtures were prepared as 1:1 molar ratios $\left(1 \times 10^{-3} \mathrm{M}\right.$ each $)$ in $\mathrm{H}_{2} \mathrm{O}$, and nebulized. A blank sample was run between each sample to ensure zero cross contamination. Nebulized particles were passed through a silica drying column, and sampled into the mass spectrometer. The air flow rate of the nebulizer was maintained at 1.26 $\mathrm{L} / \mathrm{min}$ which kept the particle stream below an average rate of 1.5 per s, well within the linear regime of the instrument [23]. Peptide powder aerosols were generated by gently agitating a bottle and sampling the headspace into the mass spectrometer. This gentle agitation also produced an irregular particle stream but did not exceed a rate of 1.7 particles per $\mathrm{s}$ for all powder aerosols.

\section{Mass Spectrometry}

All experiments were performed using an aerosol timeof-flight mass spectrometer (ATOFMS) instrument [23]. Briefly, particles were sampled by the instrument, at a 1 $\mathrm{L} / \mathrm{min}$ flow rate, focused into a particle stream by an aerosol nozzle, and tracked by two scattering lasers. The aerodynamic diameter of each particle was determined from its terminal velocity. This velocity determined the time at which a $266 \mathrm{~nm}$ Nd:YAG desorption/ ionization laser was fired at each particle. Therefore, each tracked particle was also fired on by the ionization laser. Positive and negative mass spectra were obtained via two TOF reflectron mass analyzers. All spectra were acquired at a laser pulse energy of $0.21 \mathrm{~mJ}$ per pulse, which was optimized for Bacillus spore spectra. The Nd:YAG laser diameter has been previously measured at $400 \mathrm{um}$ [24], thus $0.21 \mathrm{~mJ}$ corresponds to an average fluence of $\sim 1.7 \mathrm{~nJ} / \mu \mathrm{m}^{2}$ [24]. All spectra shown are the sum averages of single particle spectra for each sample.

\section{Effective Ionization Probability}

There are two possibilities that occur for tracked particles. Some particles tracked and fired upon by the ionization laser generate a mass spectrum, while other particles do not generate a mass spectrum. Effective ionization probability (EIP) was calculated as the number of particles that generate a mass spectrum divided by all tracked particles within the size range of $0.3 \mu \mathrm{m}$ to $3.0 \mu \mathrm{m}$. Since particles of similar size experience similar divergence, defining a consistent size range effectively removes particle divergence bias from the EIP values. All samples were run in triplicate in order to generate standard deviation error bars shown on all the EIP plots. All instrument conditions affecting EIP values, such as the scattering laser positions, the ionization laser position, spectrum detection threshold etc., were held constant throughout this study.

\section{Results and Discussion}

The BAMS mass spectra of Bacillus spores contain primarily analytes from the spore's core including DPA, arginine and glutamic acid [10,12, 13]. These conclusions were reached after growing the organism in ${ }^{13} \mathrm{C}$ and ${ }^{15} \mathrm{~N}$ enriched media. The appropriate mass shifts obtained in each media along with the mass yielded the identity of the amino acids and dipicolinic acid obtained in the spectra. To understand better why only core components are observed in the spore spectra, a series of standard amino acid and DPA bio-aerosols were analyzed by BAMS under conditions that coincide with those used for Bacillus spores. There are a number of factors that govern the appearance of a mass spectrum for a tracked particle. Many of these are physical 

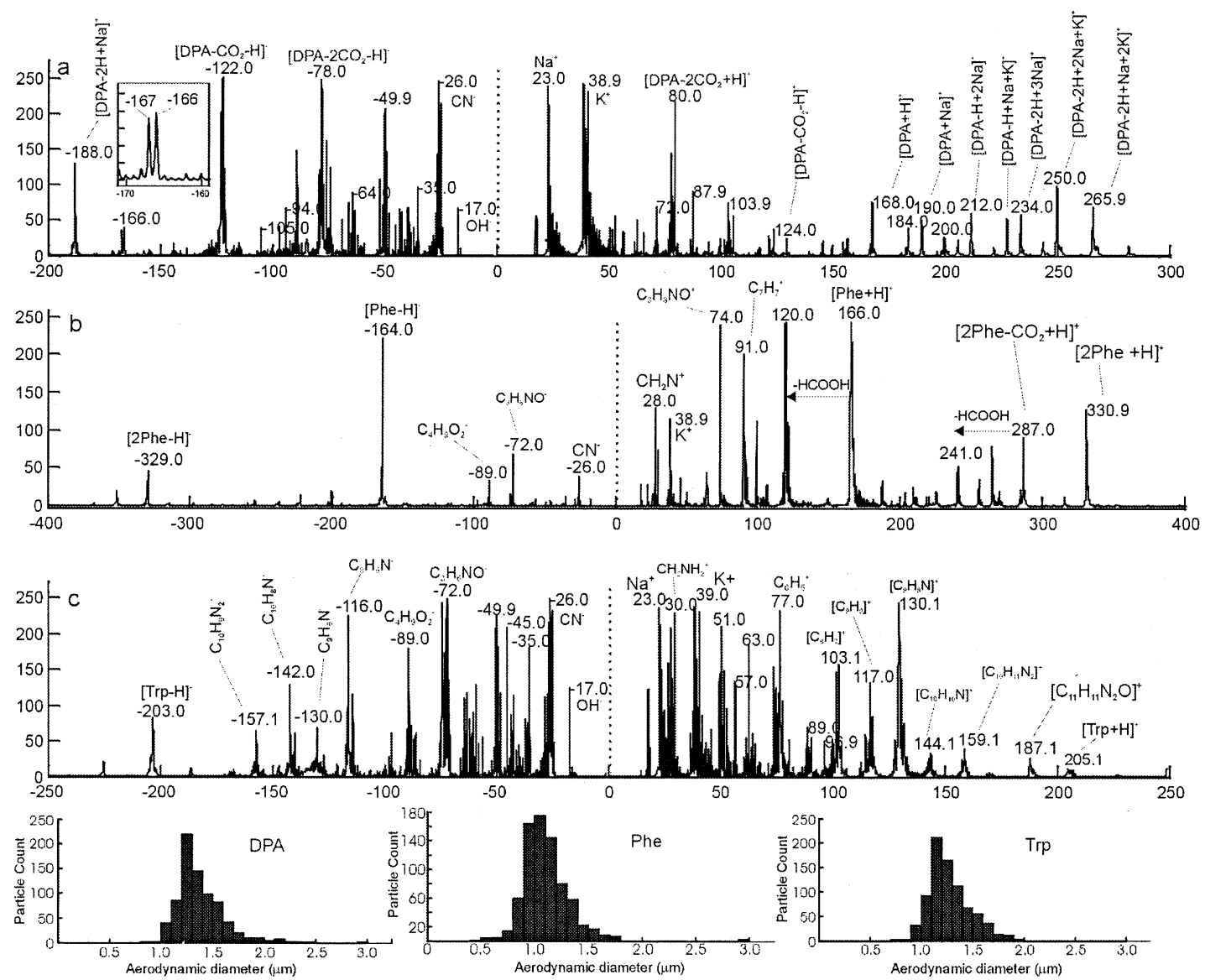

Figure 1. (a) Average of 770 single particle dipicolinic acid dual polarity spectra. (b) Average of 760 single particle phenyl alanine dual polarity spectra. (c) Average of 799 single particle tryptophan dual polarity spectra. Size histograms of all bio-aerosol particles used to generate the average spectra for each standard are shown as well. All spectra were acquired at $0.21 \mathrm{~mJ}$ per pulse. All amino acid and DPA particles were aerosolized from $2 \times 10^{-3} \mathrm{M}$ solutions via a Collison nebulizer.

factors such as the divergence of the particle beam and the interaction between the particle and the ionizing laser. A significant factor is the diameter and the profile of the Nd:YAG laser beam. In these experiments, the diameter is approximately $400 \mu \mathrm{m}$ [24]. Because the profile is Gaussian, the ionization of the particle will depend on where it interacts with the beam. There are areas in the beam that have high fluence, where the probability of ionization is high. Given that particles of the same size will experience similar divergence, we can extract the chemical factors by comparing the ionization of particles of different composition. We chose a narrow size distribution corresponding to the region of 0.3 to 3 $\mu \mathrm{m}$ where the divergence characteristics were similar. We define an EIP, which is the ratio $(\times 100)$ of tracked particles that produce mass spectra to the total number of particles that are tracked within 0.3 to $3.0 \mu \mathrm{m}$. A value of $100 \%$ means that all tracked particles produce mass spectra. These conditions were not attainable due to the factors such as particle beam divergence and the specific interaction of the particles with the various regions of the laser beam, as described earlier. More typical values ranged from zero to approximately $40 \%$.

\section{Analyses of Aerosolized Single Component Particles}

Particles of pure dipicolinic acid yielded the highest EIP $(35 \%)$ of all pure particles. Figure 1a shows a representative spectrum of DPA (averaged from 770 single particle spectra) in which both protonated and deprotonated molecular ions were observed at $\mathrm{m} / \mathrm{z}+168.0$ and -166.0 , respectively. Note that both positive and negative modes are presented in a single spectrum. For clarity cations will have positive $\mathrm{m} / \mathrm{z}$ values while anions will have negative $m / z$ values. The molecular ion region in the anion mode is noteworthy as both a deprotonated $(\mathrm{m} / \mathrm{z}-166.0)$ and what appears to be a negative molecular ion $(m / z-167.0)$, due presumably to an electron capture event, are observed. This electron capture species has also been observed in Bacillus spore spectra [13]. Fragments of the protonated and deprotonated molecular ions via $\mathrm{CO}_{2}$ losses were also observed at $m / z+124.0$ and +80.0 in the positive mode, and $\mathrm{m} / \mathrm{z}$ -122.0 and -78.0 in the negative mode. A series of potassiated and sodiated species were observed at $\mathrm{m} / \mathrm{z}$ +190.0, +212.0, +228.0, +234.0, +250.0, and +265.9. A 

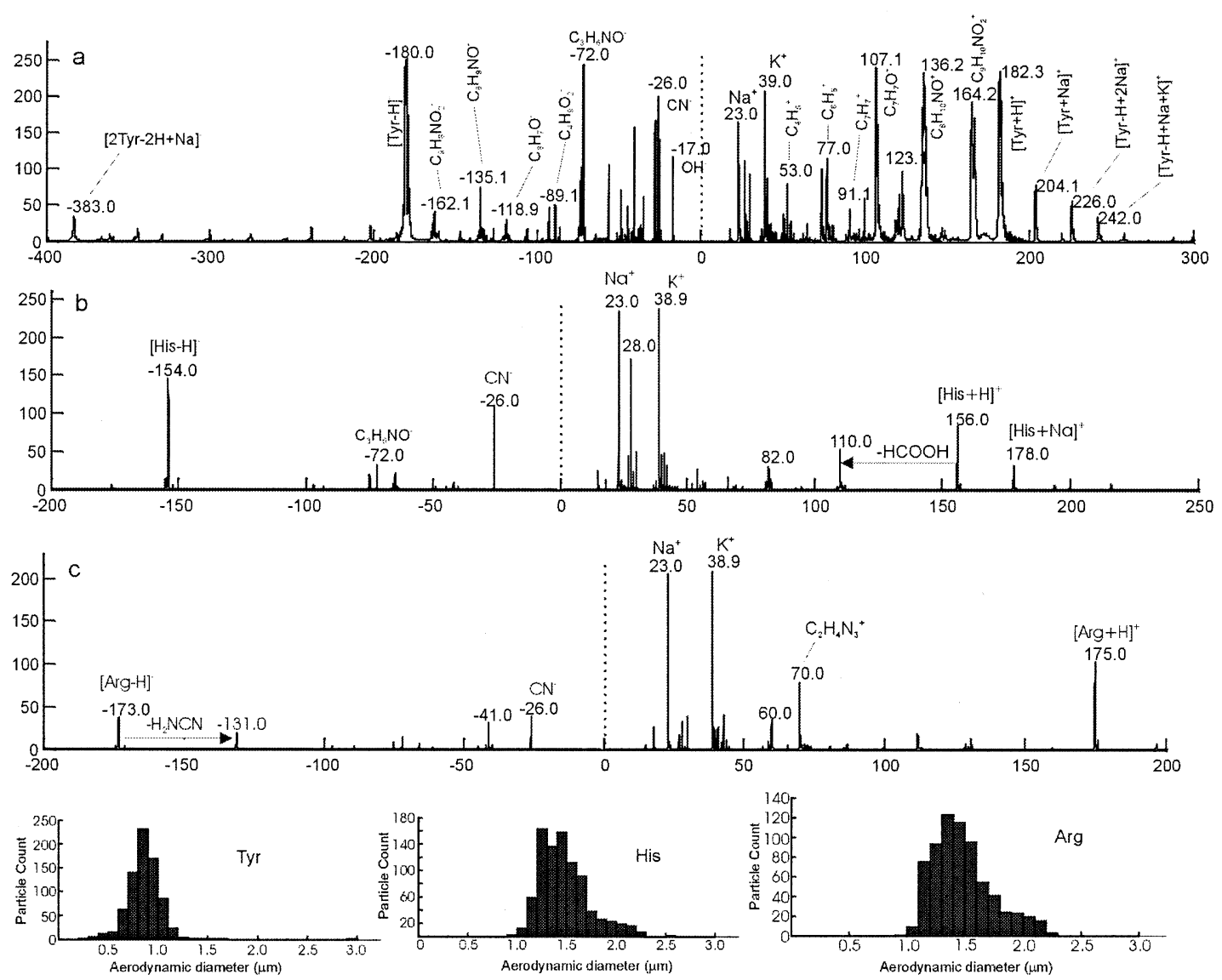

Figure 2. (a) Average of 744 tyrosine single particle dual polarity spectra. (b) Average of 863 single particle histidine dual polarity spectra. (c) Average of 552 single particle arginine dual polarity spectra. Size histograms of all bio-aerosol particles used to generate the average spectra for each standard are shown as well. All spectra were acquired at $0.21 \mathrm{~mJ}$ per pulse. All amino acid and DPA particles were aerosolized from $2 \times 10^{-3} \mathrm{M}$ solutions via a Collison nebulizer.

sodiated species was also observed in the negative spectrum at $m / z-188.0$.

Particles of phenyl alanine had a similarly high EIP of $31 \%$. The spectrum (average of 760 single particle spectra) is shown in Figure 1b. Both protonated and deprotonated molecular ions were observed at $\mathrm{m} / \mathrm{z}$ +166.0 and -164.0 , respectively. Unlike DPA, the negative electron capture molecular ion was not observed. Extensive fragmentation yielded a series of carbocations including losses of side chains and a carboxylic acid group. The protonated dimer at $\mathrm{m} / z+330.9$ as well as fragments of this dimer were observed. $\mathrm{A} \mathrm{CO}_{2}$ loss at $m / z+287.0$ and $\mathrm{H}_{2} \mathrm{CO}_{2}$ loss at $m / z+241.0$ were both present. The presence of the dimer and its associated fragments indicate that the interaction in the protonbound dimer is strong.

The spectrum for tryptophan (average of 799 single particle spectra), another aromatic amino acid, is shown in Figure 1c. The EIP for these particles was relatively high at $30 \%$. Both protonated and deprotonated molecular ions were observed at $\mathrm{m} / \mathrm{z}+205.1$ and -203.0 , respectively. A fragment ion due to the loss of water was observed at $m / z+187.1$. Fragment ions containing the side chain were observed in the negative spectrum at $m / z-157.0,-142.0,-130.0$, and -116.0 . A complementary fragment consisting of only the main chain was observed at $m / z-72.0$. Fragments were also observed in the positive mode at $\mathrm{m} / \mathrm{z}+159.1,+144.1$, $+130.1,+117.0,+103.1,+77.0$, and +30.0. The tryptophan spectra contained a high degree of fragmentation, similar to that seen in DPA.

Tyrosine had a lower EIP value (23\%) compared to the other aromatic amino acids. The spectrum (average of 744 single particle spectra) shown in Figure 2a contained both protonated and deprotonated molecular ions at $m / z+182.3$ and -180.0 , respectively. Losses of water from both molecular ions were observed at $\mathrm{m} / \mathrm{z}$ +164.2 and -162.1 . A deprotonated dimer was observed with one hydrogen replaced by a sodium at $\mathrm{m} / \mathrm{z}$ -383.0. Deprotonated fragments containing the side chain were observed in the negative ion spectra at $\mathrm{m} / \mathrm{z}$ -135.1 and -118.9 . Deprotonated fragments containing the main chain were observed at $m / z-89.0$ and -72.0 , as well. The corresponding fragments in the positive mode were observed at $m / z+165.9,+136.2,+107.1$, $+91.1,+77.0$, and +53.0. 


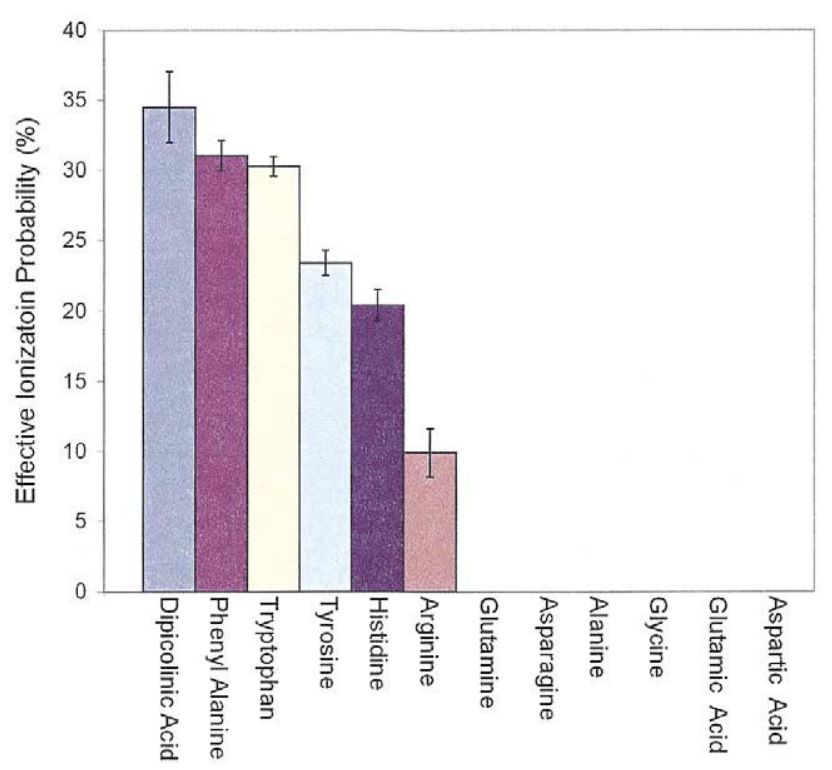

Figure 3. Plot of EIP values for all amino acid and dipicolinic acid aerosols. EIP values were calculated as a percentage of particles that generated mass spectra divided by the total number of particles tracked within $0.3-3.0 \mu \mathrm{m}$. Error bars represent the EIP standard deviations of each sample run in triplicate. Laser pulse energy was set to $0.21 \mathrm{~mJ}$ per pulse for all samples.

Histidine also had a lower EIP of $20 \%$. Figure $2 b$ shows the mass spectrum (average of 863 single particle spectra) with both protonated and deprotonated molecular ions at $m / z+156.0$ and -154.0 , respectively. A sodiated quasimolecular ion was observed in the positive mode at $\mathrm{m} / \mathrm{z}+178.0$. Limited fragmentation was observed, via a loss of $\mathrm{HCOOH}$ to $\mathrm{m} / z+110.0$, and a main chain fragment at $m / z-72.0$.

The basic amino acid arginine, had no aromatic side chain group, but had an EIP of $10 \%$. Figure $2 \mathrm{c}$ shows the mass spectrum (average of 552 single particle spectra) in which protonated and deprotonated molecular ions were observed at $m / z+175.0$ and -173.0 , respectively. Despite it being the most basic amino acid, an abundant deprotonated molecular ion was observed. Fragmentation along the side chain yielding a loss of $\mathrm{H}_{2} \mathrm{NCN} \mathrm{m} / \mathrm{z}$ -131.1 was observed. A fragment containing primarily the side chain was also observed at $m / z+70.0$.

The EIP values of dipicolinic acid and the amino acids that were investigated are plotted in Figure 3. High EIP values are clearly associated with the presence of a UV absorbing aromatic side chain. These results are consistent with previous findings for PAHs and other aromatic aerosols [25]. Glutamic acid, aspartic acid, alanine, glutamine, glycine, and asparagine were also examined but had EIP values of zero as no mass spectra were obtained for these particles at a laser pulse energy of $0.21 \mathrm{~mJ}$ per pulse. The presence of glutamic acid in Bacillus spore spectra may be due to the large amount of DPA in the spore core. This naturally occurring matrix may aid in the ionization of non-absorbing components.

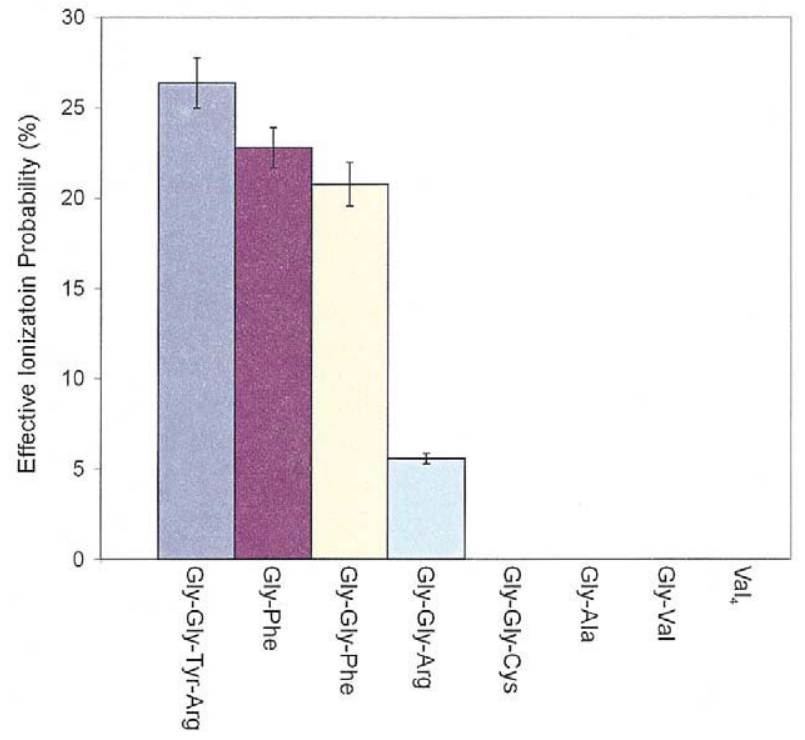

Figure 4. Plot of EIP values of all peptide dry powder aerosols. EIP values were calculated as a percentage of particles that generated mass spectra divided by the total number of particles tracked within 0.3-3.0 $\mu \mathrm{m}$. Error bars represent the EIP standard deviations of each sample run in triplicate. Laser pulse energy was set to $0.21 \mathrm{~mJ}$ per pulse for all samples.

\section{Analyses of Single Component Particles of Peptides}

A similar study of small peptides was performed in order to determine if peptides containing UV absorbing aromatic amino acid residues had significantly higher EIP values than those without. The EIP values of the various peptides are summarized in Figure 4. A similar trend was observed in which peptides containing a phenyl group had significantly higher EIP values than those without. Gly-Gly-Tyr-Arg, which contains both an aromatic amino acid residue and a basic residue, had the highest EIP value at 26\%. Gly-Gly-Phe and Gly-Phe had EIP values that were slightly less at 23 and $21 \%$, respectively. Peptides that did not contain aromatic amino acids had the lowest EIP values at less than $6 \%$. These results were again consistent with efficient ionization of UV absorbing aromatic amino acid aerosols, and substituted aromatic compounds ionized from a surface as in MALDI [26].

The mass spectrum for Gly-Phe, shown in Figure 5a, contained the protonated and deprotonated molecular ions at $m / z+223.1$ and -221.1 , respectively. Fragments resulting from the loss of $\mathrm{CO}_{2}$, glycine, and $\mathrm{HCOOH}$ from the protonated molecular ion were also observed in the positive spectrum at $\mathrm{m} / \mathrm{z}+179.1,+166.1$, and +120.0 , respectively. A deprotonated molecular ion dimer and a corresponding water loss were observed, albeit with relatively low abundances, at $\mathrm{m} / \mathrm{z}-443.3$ and -425.3 , respectively.

Figure $5 b$ shows the spectra of Gly-Gly-Phe, which contained both protonated and deprotonated molecular ions at $m / z+280.1$, and -278.1 , respectively. Successive losses of glycine residues with $\mathrm{HCOOH}$ were observed 

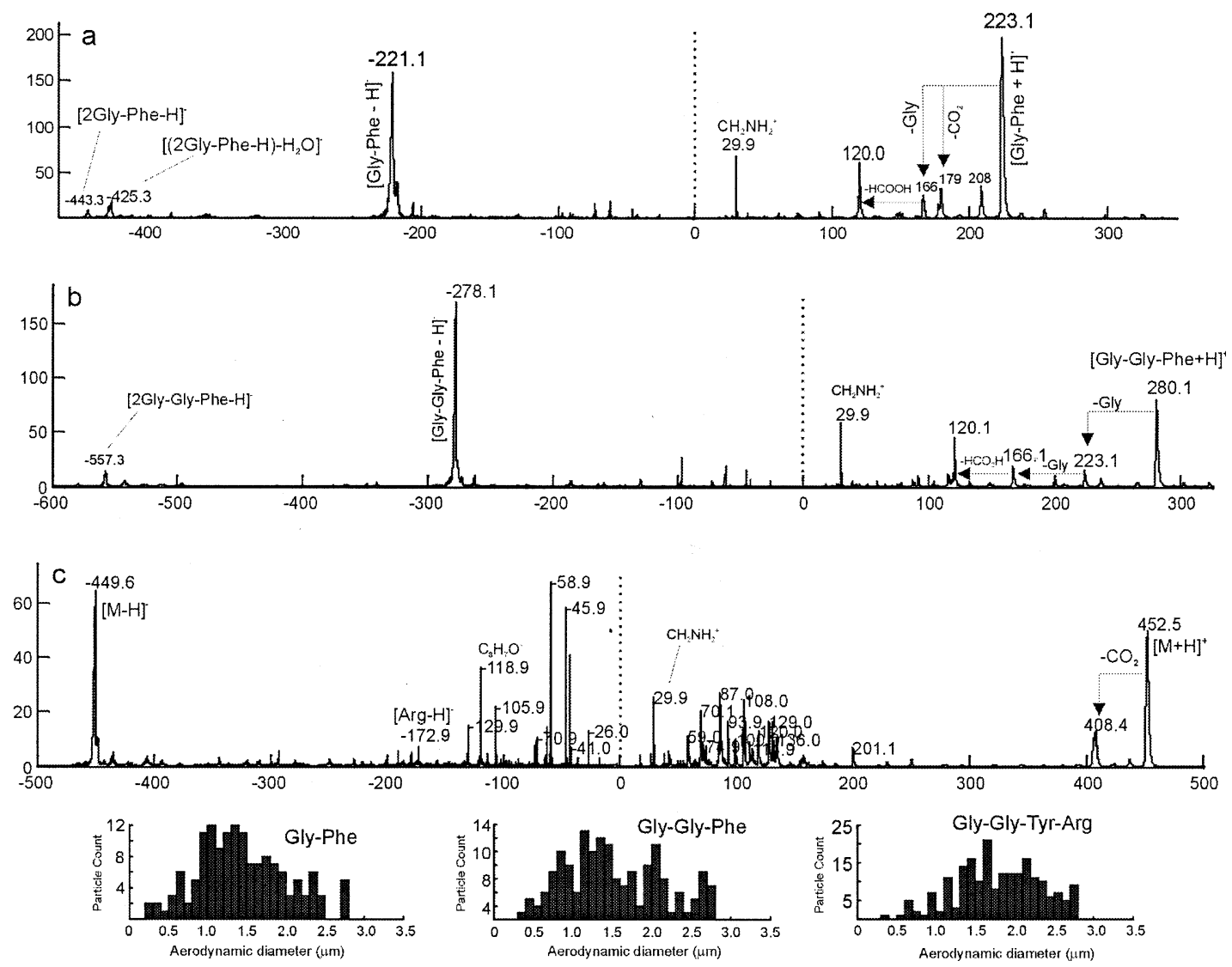

Figure 5. (a) Average of 144 single particle Gly-Phe dual polarity spectra. (b) Average of 151 single particle Gly-Gly-Phe dual polarity spectra. (c) Average of 200 single particle Gly-Gly-Tyr-Arg dual polarity spectra. Size histograms of all bio-aerosol particles used to generate the average spectra for each standard are shown as well. All spectra were acquired at $0.21 \mathrm{~mJ}$ per pulse. All peptide particles were aerosolized from dry powder.

at $m / z+223.1,+166.1$, and +120.0 . A deprotonated molecular ion dimer was also observed, in relatively low abundance, at $m / z-557.3$. The presence of molecular ion dimers in phenyl alanine containing peptides was consistent with dimers observed for the Phe amino acid.

The spectra of Gly-Gly-Tyr-Arg, shown in Figure 5c, contained both protonated and deprotonated molecular ions at $\mathrm{m} / \mathrm{z}+452.5$, and -449.6 . Fragmentation, via a $\mathrm{CO}_{2}$ loss from the protonated molecular ion to $\mathrm{m} / \mathrm{z}$ +408.4, was observed in the positive mode. Although MALDI has been pursued for enhanced ionization of peptide aerosols [14-19], the results presented in this report show self-contained aromatic residues are sufficient for direct desorption/ionization of small peptides with a $266 \mathrm{~nm}$ laser.

\section{Component-Assisted Ionization in Mixtures}

The presence of an absorbing medium in the particle is clearly necessary to enhance the ionization of the particle. Whether the absorbing medium can similarly enhance the ionization of a secondary component, akin to a gas-phase matrix-assisted ionization (as in

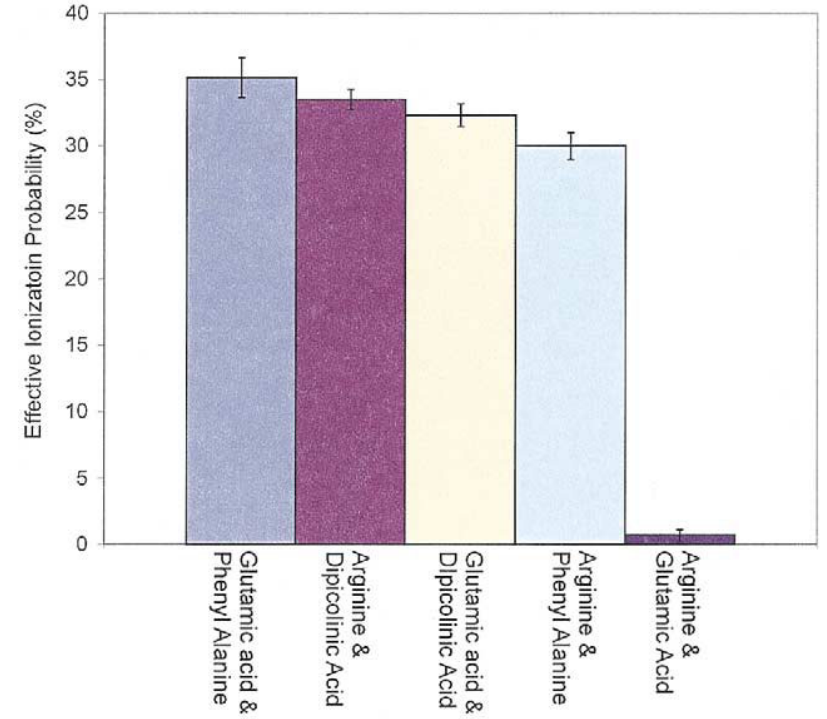

Figure 6. Plot of EIP values of glutamic acid, arginine, dipicolinic acid, and phenyl alanine mixtures. EIP values were calculated as a percentage of particles that generated mass spectra divided by the total number of particles tracked within $0.3-3.0 \mu \mathrm{m}$. Error bars represent the EIP standard deviations of each sample run in triplicate. Laser pulse energy was set to $0.21 \mathrm{~mJ}$ per pulse for all samples. 

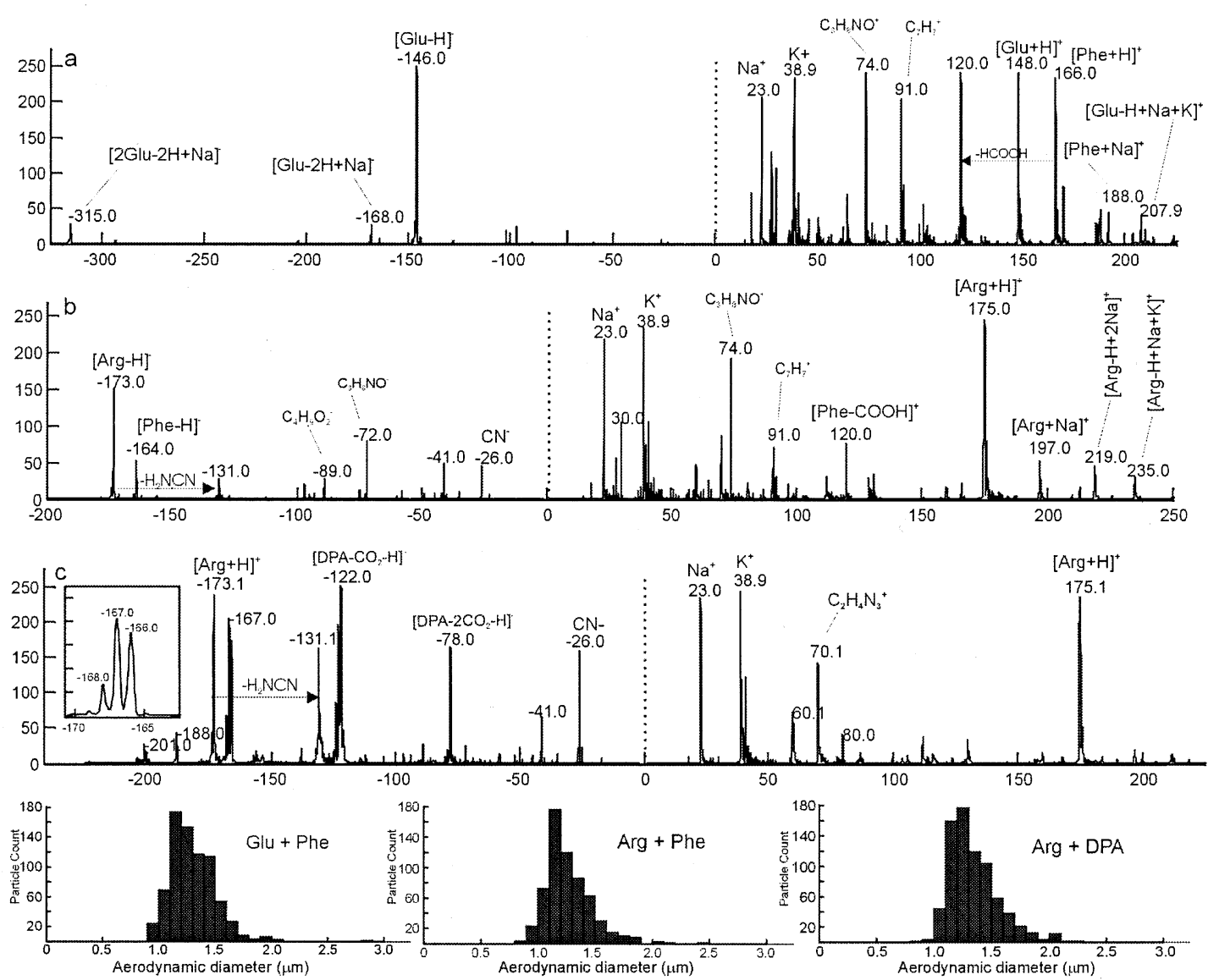

Figure 7. (a) Average of 751 single particle phenyl alanine/glutamic acid dual polarity spectra. (b) Average of 610 single particle phenyl alanine/arginine dual polarity spectra. (c) Average of 752 DPA/arginine single particle dual polarity spectra. Size histograms of all bio-aerosol particles used to generate the average spectra for each standard are shown as well. All spectra were acquired at $0.21 \mathrm{~mJ}$ per pulse. All mixture particles were aerosolized from 1:1 molar solutions $\left(1 \times 10^{-3} \mathrm{M}\right.$ each $)$ via a Collison nebulizer.

MALDI), was investigated by studying a series of mixtures. Ion suppression is also known to occur in many MALDI experiments due to secondary ion plume proton transfer reactions governed by relative gas phase basicities [20-22]. The possibility of a similar phenomenon occurring in the ionization of bio-aerosols was investigated by mixing components with differing acid/base properties and observing both positive and negative spectra simultaneously. A nebulized aerosol begins as a liquid droplet. This droplet then passes through a silica drying tube in which the particle dries down. The composition of the dried particle is assumed to reflect that of the bulk solution. Thus when the particle dries down, a 1:1 molar ratio solution will produce a 1:1 molar ratio solid particle. This allows for the investigation of suppression effects in particles with defined compositions.

Dipicolinic acid was mixed with two amino acids with differing gas-phase basicities, namely arginine and glutamic acid. The mixtures were prepared as 1:1 molar solutions and nebulized. The EIP values of various mixtures are summarized in Figure 6. For comparison, mixtures with Phe were also examined. The addition of aromatic components yielded higher EIP values for the amino acids that did not contain aromatic groups. For example, Arg increased from 10 to 30 and 33\% when mixed with Phe and DPA, respectively. Similarly, Glu, which yielded no spectra as a pure compound, also had higher EIP values of 32 and 35\% when mixed with DPA and Phe, respectively. Note however, when Arg and Glu were mixed, the EIP did not increase and even decreased relative to pure Arg. The results from the mixture studies suggest a MALDI-like effect whereby the ionization of the amino acids such as Arg, Glu, etc. were enhanced by the presence of DPA and Phe. These results help explain the presence of non-aromatic components in the Bacillus spore spectra. The high amount of DPA in the spore core may indeed aid in the ionization of non-aromatic components.

Although efficient absorption of the radiation by the particle is a requisite for desorption and ionization, the appearance of ionic species may also be governed by ion-molecule reactions in the nascent plume. The typical spectrum (average of 751 single particle spectra) of a 


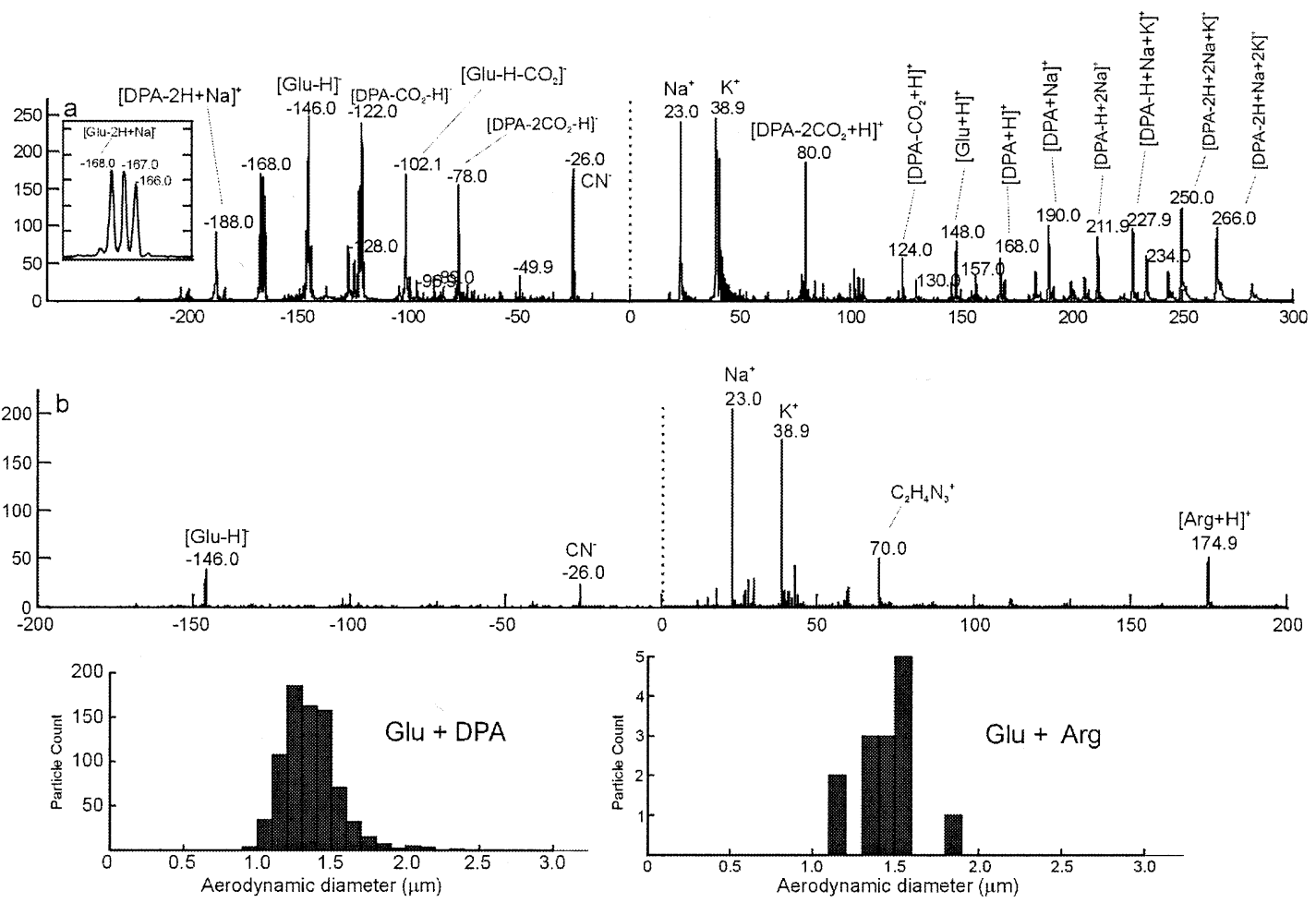

Figure 8. (a) Average of $784 \mathrm{DPA} /$ glutamic acid single particle dual polarity spectra. (b) Average of 14 arginine/glutamic acid single particle dual polarity spectra. Size histograms of all bio-aerosol particles used to generate the average spectra for each standard are shown as well. All spectra were acquired at $0.21 \mathrm{~mJ}$ per pulse. All mixture particles were aerosolized from 1:1 molar solutions $(1 \times$ $10^{-3} \mathrm{M}$ each) via a Collison nebulizer.

Glu plus Phe mixture is shown in Figure 7a. The positive mode contained both protonated molecular ions at $m / z+166.0$ and +148.0 , respectively. Fragments of Phe were also observed at $\mathrm{m} / \mathrm{z}+120.0,+91.0$, and +74.0 . The negative mode was dominated by deprotonated glutamic acid at $m / z-146.0$ with little or no trace of deprotonated phenylalanine. Deprotonated glutamic acid where the second carboxylic acid hydrogen was replaced by sodium was observed at $m / z-168.0$, while a deprotonated dimer bound by a single $\mathrm{Na}^{+}$at $\mathrm{m} / \mathrm{z}$ -315.0 was also observed. In the pure Phe spectra, the deprotonated species was a dominant peak but is completely absent in the mixture. This is consistent with the differences in gas phase basicity of each component (Phe: 888.9 kJ/mole, Glu: 879.1 kJ/mole).

This suppressive effect was also observed in the spectrum of an arginine and phenyl alanine mixture, shown in Figure $7 \mathrm{~b}$. While the negative spectrum contained both deprotonated molecular ions at $m / z-173.0$ and -164.0 for Phe and Arg, respectively, protonated arginine with $\mathrm{m} / \mathrm{z}+175.0$ dominates the positive mode. This result is also consistent with the gas phase basicities of the two compounds (Arg: $1007 \mathrm{~kJ} / \mathrm{mole}$, Phe: $888.9 \mathrm{~kJ} /$ mole).

Similar observations were made when Arg was mixed with DPA. The positive spectrum is again dominated by protonated arginine at $\mathrm{m} / \mathrm{z}+175.0$ as shown in Figure 7c. A low abundance fragment of arginine was seen at $\mathrm{m} / z+70.0$. The only evidence of DPA in the positive spectrum was a low abundance [DPA $-2 \mathrm{CO}_{2}$ $+\mathrm{H}^{+}$fragment at $\mathrm{m} / \mathrm{z}+80.0$. The negative mode contained both deprotonated molecular ions of arginine and dipicolinic acid at $m / z-173.0$ and -166.0 , respectively. Fragmentation of arginine, via a side chain loss, was observed at $m / z-131.0$. Fragmentation of DPA, via $\mathrm{CO}_{2}$ losses, was observed at $\mathrm{m} / \mathrm{z}-122.0$ and -78.0 , which was also seen in pure DPA. An odd electron DPA species was also observed in the negative spectrum at $m / z-167.0$, which is also seen in pure DPA.

Mixing DPA and glutamic acid, two acidic components, produced spectra shown in Figure 8a that lacked a dominant component in either mode. Both protonated and deprotonated DPA species were observed at $\mathrm{m} / \mathrm{z}$ +168.0 , and -166.0 , respectively. Glutamic acid was also present in both protonated and deprotonated forms at $m / z+148.0$ and -146.0 ., respectively. Protonated and deprotonated fragments of DPA, via $\mathrm{CO}_{2}$ losses, were observed at $m / z+124.0,+80.0,-122.0$, and -78.0 , respectively. A glutamic acid fragment, via a $\mathrm{CO}_{2}$ loss, was also observed, at $\mathrm{m} / z-102.1$. Various sodiated and potassiated quasimolecular ions were observed from DPA at $m / z+266.0,+250.0,+227.9$, $+211.9,+190.0$, and -188.0 . A sodiated glutamic acid quasimolecular ion was also observed at $m / z-168.0$.

Mixing glutamic acid and arginine, an acidic and basic amino acid with no aromatic side-chain, yielded a 
lower EIP. However, the spectra (Figure 8b) provide further evidence of acid/base proton transfer competition in the ion plume. The positive spectrum contains only a protonated arginine molecular ion at $m / z+175.0$, and a fragment at $\mathrm{m} / z+70.0$. The negative spectrum contains only the deprotonated glutamic acid molecular ion at $m / z-146.0$.

\section{Conclusions}

The EIP of micrometer bio-aerosol particles is significantly higher when an absorbing residue is present. Mixing absorbing and non-absorbing compounds provides a MALDI-like environment that increases the EIP of the non-absorbing molecule. The ions observed in each polarity were found to be dependent on the relative acidities and basicities of the components in the mixture. The presence of protonated DPA and Phe in the single component particles but not in mixtures with Arg indicate a competition that likely occurs during the desorption/ionization process, a process similar to that believed for MALDI [20,27]. These results also explain a number of observations in the analyses of the spores. The presence of DPA in the Bacillus spore core likely contributes to the ionization process allowing the observation of amino acids within the core that do not have UV absorbing aromatic side chains.

Generation of biomarker ions from spores and other cells via BAMS involves a two step process of desorption and ionization. The correlation of high EIP values with UV absorbing aromatic chromophores such as DPA is indicative of an enhanced desorption process in which the laser energy is efficiently coupled with the analyte. Ionization is likely affected by proton transfer reactions in the plume yielding protonated and deprotonated ions which were observed in positive and negative polarities, respectively. This two stage process explains the presence of peaks in the spore spectra from non-absorbing analytes such as arginine and glutamic acid. Future biomarkers will need to contain aromatic residues such as DNA, RNA, and peptides or be in the presence an absorbing chromophore that acts as a natural matrix.

\section{Acknowledgments}

This work was performed under the auspices of the U.S. Department of Energy by University of California Lawrence Livermore National Laboratory under contract W-7405-ENG-48. This project was funded by the Technical Support Working Group of the Department of Defense. SR and GC acknowledge the LLNL Student Employee Graduate Research Fellowship (SEGRF) for support.

\section{References}

1. Anhalt, J. P.; Fenselau, C. Identification of Bacteria Using Mass Spectrometry. Anal. Chem. 1975, 47, 219-225.
2. Heller, D. N.; Cotter, R. J.; Fenselau, C. Profiling of Bacteria by Fast Atom Bombardment Mass Spectrometry. Anal. Chem. 1987, 59, 2806-2809.

3. Heller, D. N.; Murphy, C.; Cotter, R. J.; Fenselau, C.; Uy, O. M. Constant Neutral Loss Scanning for the Characterization of Bacterial Phospholipids Desorbed by Fast Atom Bombardment. Anal. Chem. 1988, 60, 2787-2791.

4. Fenselau, C.; Demirev, P. A. Characterization of Intact Microorganisms by MALDI Mass Spectrometry. Mass Spectrom. Rev. 2001, 20, 157-171.

5. Claydon, M. A.; Davey, S. N.; Jones, V. E.; Gordon, D. B. The Rapid Identification of Intact Microorganisms Using Mass Spectrometry. Nat. Biotechnol. 1996, 14, 1584-1586.

6. Despeyroux, D.; Phillpotts, R.; Watts, P. Electrospray Mass Spectrometry for Detection and Characterization of Purified Cricket Paralysis Virus (CrPV). Rapid Commun. Mass Spectrom. 1996, 10, 937-941.

7. Holland, R. D.; Wilkes, J. G.; Rafii, F.; Sutherland, J. B.; Persons, C. C.; Voorhees, K. J.; Jay, J. O. Rapid Identification of Intact Whole Bacteria Based on Spectral Patterns Using Matrix-Assisted Laser Desorption/Ionization with Time-of-Flight Mass Spectrometry. Rapid Commun. Mass Spectrom. 1996, 10, 1227-1232.

8. Krishnamurthy, T.; Ross, P. L.; Rajamani, U. Detection of Pathogenic and Non-Pathogenic Bacteria by Matrix-Assisted Laser Desorption/Ionization Time-of-Flight Mass Spectrometry. Rapid Commun. Mass Spectrom. 1996, 10, 883-888.

9. Ullom, J. N.; Frank, M.; Gard, E. E.; Horn, J. M.; Labov, S. E.; Langry, K.; Magnotta, F.; Stanion, K. A.; Hack, C. A.; Benner, W. H. Discrimination Between Bacterial Spore Types Using Time-of-Flight Mass Spectrometry and Matrix Free Infrared Laser Desorption and Ionization. Anal. Chem. 2001, 73, 23312337.

10. Fergenson, D. P.; Pitesky, M. E.; Tobias, H. J.; Steele, P. T.; Czerwieniec, G. A.; Russell, S. C.; Lebrilla, C. B.; Horn, J. M.; Coffee, K. R.; Srivastava, A.; Pillai, S. P.; Shih, M.; Hall, H. L.; Ramponi, A. J.; Chang, J. T.; Langlois, R. G.; Estacio, P. L.; Hadley, R. T.; Frank, M.; Gard, E. E. Reagentless Identification of Individual Bioaerosol Particles in Milliseconds. Anal. Chem. 2003, 76, 373-378.

11. Higgins, J. A. Annals of the New York Academy of Sciences 1999, pp 130-148.

12. Gould, G. W.; Hurst, A. The Bacterial Spore; Academic Press: London/New York, 1969, pp 249-263.

13. Czerwieniec, G.; Russell, S. C.; Tobias, H. J.; Fergenson, D. P.; Steele, P. T.; Pitesky, M. E.; Horn, J. M.; Frank, M.; Gard, E. E.; Lebrilla, C. B. unpublished results.

14. Mansoori, B. A.; Johnston, M. V.; Wexler, A. S. Matrix-Assisted Laser Desorption/Ionization of Size- and Composition-Selected Aerosol Particles. Anal. Chem. 1996, 68, 3595-3601.

15. Jackson, S. N.; Murray, K. K. Matrix Addition by Condensation for Matrix-Assisted Laser Desorption/Ionization of Collected Aerosol Particles. Anal. Chem. 2002, 74, 4841-4844.

16. Stowers, M. A.; van Wuijckhuijse, A. L.; Marijnissen, J. C. M.; Scarlett, B.; van Baar, B. L. M.; Kientz, C. E. Application of Matrix-Assisted Laser Desorption/Ionization to On-Line Aerosol Time-of-Flight Mass Spectrometry. Rapid Commun. Mass Spectrom. 2000, 14, 829-833.

17. Murray, K. M.; Russell, D. H. Aerosol Matrix-Assisted Laser Desorption Ionization Mass Spectrometry. J. Am. Soc. Mass Spectrom. 1994, 5, 1-9.

18. Fei, X.; Wei, G.; Murray, K. K. Aerosol MALDI with a Reflectron Time-of-Flight Mass Spectrometer. Anal. Chem. 1996, 68, 1143-1147.

19. He, L.; Murray, K. K. Three Hundred Thirty-Seven nm MatrixAssisted Laser Desorption/Ionization of Single Aerosol Particles. J. Mass Spectrom. 1999, 34, 909-914. 
20. Knochenmuss, R.; Stortelder, A.; Breuker, K.; Zenobi, R. Secondary Ion-Molecule Reactions in Matrix-Assisted Laser Desorption Ionization. J. Mass Spectrom. 2000, 35, 1237-1245.

21. Knochenmuss, R.; Karbach, V.; Wiesli, U.; Breuker, K.; Zenobi, R. The Matrix Suppression Effect in Matrix-Assisted Laser Desorption/Ionization: Application to Negative Ions and Further Characteristics. Rapid Commun. Mass Spectrom. 1998, 12, 529-534.

22. Stevenson, E.; Breuker, K.; Zenobi, R. Internal Energies of Analyte Ions Generated from Different Matrix-Assisted Laser Desorption/Ionization Matrices. J. Mass Spectrom. 2000, 35, 1035-1041.

23. Gard, E. E.; Mayer, J. E.; Morrical, B. D.; Dienes, T.; Fergenson, D. P.; Prather, K. A. Real-Time Analysis of Individual Atmospheric Aerosol Particles-Design and Performance of a Portable ATOFMS. Anal. Chem. 1997, 69, 4083-4091.
24. Steele, P. T.; Tobias, H. J.; Fergenson, D. P.; Pitesky, M. E.; Horn, J. M.; Czerwieniec, G.; Russell, S. C.; Lebrilla, C. B.; Gard, E. E.; Frank, M. Laser Power Dependence of Mass Spectral Signatures from Individual Bacterial Spores for Bioaerosol Mass Spectrometry. Anal. Chem. 2003, 75, 54805787.

25. Noble, C. A.; Prather, K. A. Single Particle Characterization of Albuterol Metered Dose Inhaler Aerosol in Near Real-Time. Aerosol Sci. Technol. 1998, 29, 294-306.

26. Gimon-Kinsel, M.; Preston-Schaffter, L. M.; Kinsel, G. R.; Russell, D. H. Effects of Matrix Structure/Acidity on Ion Formation in Matrix-Assisted Laser Desorption Ionization Mass Spectrometry. J. Am. Chem. Soc. 1997, 119, 2534-2540.

27. Karas, M.; Gluckmann, M.; Schafer, J. Ionization in MatrixAssisted Laser Desorption/Ionization. J. Mass Spectrom. 2000, $35,1-12$. 